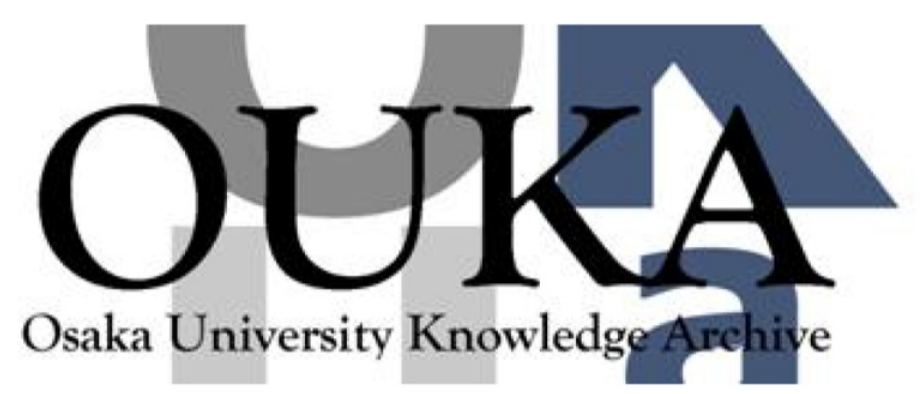

\begin{tabular}{|c|c|}
\hline Title & $\begin{array}{l}\text { Imaging of local stiffness of damaged } \\
\text { polycrystalline copper : Nondestructive } \\
\text { evaluation by resonance ultrasound microscopy }\end{array}$ \\
\hline Author (s) & $\begin{array}{l}\text { Ogi, Hirotsugu; Hayama, Noritaka; Niho, Hiroki } \\
\text { et al. }\end{array}$ \\
\hline Citation & $\begin{array}{l}\text { IEEE Transactions on ULtrasonics, } \\
\text { Ferroelectrics, and Frequency Control. 54(8) } \\
\text { p. 1514-p. } 1520\end{array}$ \\
\hline Issue Date & $2007-08$ \\
\hline oaire:version & AM \\
\hline URL & https://hdl. handle. net/11094/84145 \\
\hline rights & $\begin{array}{l}\text { (- } 2007 \text { IEEE. Personal use of this material is } \\
\text { permitted. Permission from IEEE must be } \\
\text { obtained for all other uses, in any current or } \\
\text { future media, including reprinting/republishing } \\
\text { this material for advertising or promotional } \\
\text { purposes, creating new collective works, for } \\
\text { resale or redistribution to servers or lists, } \\
\text { or reuse of any copyrighted component of this } \\
\text { work in other works. }\end{array}$ \\
\hline Note & \\
\hline
\end{tabular}

Osaka University Knowledge Archive : OUKA

https://ir. Library. osaka-u. ac. jp/

Osaka University 


\title{
Imaging of local stiffness of damaged polycrystalline copper: Nondestructive evaluation by resonance ultrasound microscopy
}

\author{
H. OGI, N. HAYAMA, H. NIHO, and M. HIRAO \\ Graduate School of Engineering Science, \\ Osaka University, Toyonaka, Osaka 560-8531, Japan \\ T. Morishita \\ Akashi College of Technology, Akashi, Hyogo 674, Japan*
}

(Dated: September 11, 2021)

\begin{abstract}
The distribution of the local stiffness of a polycrystalline copper exposed to a creep test was studied by resonance ultrasound microscopy. The local effective modulus was evaluated from the resonance frequency of the isolated langasite oscillator touching the specimen. Defects appeared predominantly on grain boundaries, and they were clearly visualized by the stiffness microscopy through the significant decrease of the effective stiffness. The stiffness within the grains becomes lower regardless of invisible defects. The stiffness distribution was quantitatively analyzed by the contact model between two anisotropic bodies and by the micromechanics modeling. The microscopic stiffness shows much higher sensitivity to the defects than the macroscopic stiffness.
\end{abstract}

PACS numbers: 62.20.Dc, 68.37.-d, 62.20.Hg, 81.70.Cv

*Electronic address: ogi@me.es.osaka-u.ac.jp 


\section{INTRODUCTION}

Recently, we developed resonance ultrasound microscopy (RUM) for the study of elastic properties in a local area of small-scale materials. It allowed us to evaluate the local effective elastic constant from the resonance frequency [1-4] and the local internal friction from attenuation [5] of a langasite oscillator touching the examined material. The resonance frequency and the attenuation coefficient were measured with a noncontacting matter by an antenna located near the oscillator. The oscillator was supported by a holder at the nodal points so that the mechanical contact for the support affected little the oscillator's vibration. Therefore, the oscillator was acoustically isolated from any other surrounds, and the contact with the specimen only affected the vibrational frequency and attenuation, achieving the high sensitivity and quantitative evaluation of the stiffness and damping at the local contacting area.

Here, we study the elastic stiffness distribution on creep-damaged materials with RUM for the purpose of the nondestructive evaluation. The elastic constants of a solid reflect the resistance against atomic-bond stretching and bending. They also reflect defects because defects such as voids, dislocations, and microcracks lower the effective elastic constants through the stress concentration around there. The elastic constants or sound velocities have been thus adopted for the nondestructive evaluation of damaged materials [6-8]. However, the contribution of the defects to the macroscopic stiffness is usually not so high. For example, longitudinal-wave velocities in a polycrystalline copper exposed to a creep test decrease only by $5 \%$ even in the final stage [9]. Shear-wave velocities during fatigue tests 
decrease by $2-3 \%[7,10]$ due to the dislocation multiplication. Thus, the effect of the defects on the macroscopic stiffness is normally less than $5 \%$ because of their small volume fraction in the propagation regions of the acoustic waves.

The softening due to the defects, however, must be enhanced when the measuring volume is localized because the volume fraction of the defects increases there. The RUM method can therefore be a powerful tool for detecting local damages of materials. We discuss the feasibility of the RUM method by comparing the results with the measured and calculated macroscopic stiffnesses.

\section{SPECIMENS}

The same specimens prepared in the previous study [9] were used: The material was a commercial tough pitch copper with the purity of $99.95 \%$. The sample plates for the creep test were obtained from the material. They were annealed at $800{ }^{\circ} \mathrm{C}$ for $3 \mathrm{~h}$ and then at $550{ }^{\circ} \mathrm{C}$ for $20 \mathrm{~h}$ before the creep test. The grain size is about $100 \mu \mathrm{m}$. The creep test was performed under the unidirectional stress of $6 \mathrm{MPa}$ at $500{ }^{\circ} \mathrm{C}$. The average rapture time was $597.8 \mathrm{~h}$. Three specimens were cut into 20 -mm cubes from the sample plates exposed to the creep test for $59.8,298.9$, and $538.0 \mathrm{~h}$, corresponding to $10 \%, 50 \%$, and $90 \%$ of the life. We named them specimens \#1, \#2, and \#3, respectively. The porosity was measured by the Archimedes method, and it is shown in TABLE I.

Specimen surfaces were polished by emery papers, buffed, and etched in a $5 \%$ nitric acid solution. Two surfaces perpendicular and parallel to the loading direction were scanned by 
RUM. Hence, we call the former "face A" and the latter "face B".

\section{ELASTIC IMAGING}

Physical principle of the RUM measurement can be found in the pervious studies $[3,5]$. The langasite crystal in a rectangular-parallelepiped shape with dimensions of $2 \times 2 \times 7 \mathrm{~mm}^{3}$ was used. A natural-diamond tip was attached at the center of the bottom surface of $2 \times 2$ $\mathrm{mm}^{2}$ area of the langasite crystal, which corresponds to the $\mathrm{X}$ face of the trigonal crystal. (The crystal's Young modulus along the $\mathrm{X}$ direction is the smallest, providing the highest sensitivity to the local stiffness of the specimen [5].) The resonance frequency was measured for every $10 \mu \mathrm{m}$. The biasing force, corresponding to the weight of the oscillator with the supporting holder, was $7 \mathrm{mN}$.

\section{RESULTS AND DISCUSSIONS}

Figure 1 compares images obtained by the optical microscopy (upper) and those by RUM (lower). The RUM images were constructed by a linear interpolation of the measuring points obtained every $10 \mu \mathrm{m}$. The common color-bar scale for the resonance-frequency change $(\Delta f / f)$ is used for all RUM images. The $\Delta f / f$ value changes on individual grains, reflecting the different crystallographic orientation. Some points near grain boundaries show very low $\Delta f / f$ values, indicating the presence of defects: Voids or microcracks are recognized by the optical microscopy at the region where the local stiffness significantly decreases. Some regions, however, show lower stiffness regardless of invisible defects there, indicating the presence of the defects beneath the surface. Significant observation is that the local stiffness 
inside the grains becomes lower as the creep damage advances. (The overall color in the RUM image changes from RED to ORANGE.). This means that the stiffness decreases not only near the grain boundary but also in the bulk. Thus, the RUM image more clearly shows defects through the elastic softening than does the optical microscopy.

First, we discuss the effect of the defects on the macroscopic elastic stiffness. The specimen exposed to the creep test macroscopically shows transverse (hexagonal) symmetry about the loading direction because the creep voids selectively arise on grain boundaries normal to the loading direction [9]. The specimens therefore show five independent elastic constants

$$
\left[C_{i j}\right]=\left[\begin{array}{cccccc}
C_{11} & C_{12} & C_{13} & 0 & 0 & 0 \\
C_{12} & C_{11} & C_{13} & 0 & 0 & 0 \\
C_{13} & C_{13} & C_{33} & 0 & 0 & 0 \\
0 & 0 & 0 & C_{44} & 0 & 0 \\
0 & 0 & 0 & 0 & C_{44} & 0 \\
0 & 0 & 0 & 0 & 0 & C_{66}
\end{array}\right]
$$

Hence, $C_{66}=\left(C_{11}-C_{12}\right) / 2$. Four of them $\left(C_{11}, C_{33}, C_{44}\right.$, and $\left.C_{66}\right)$ have been determined by the pulse-echo method [9], and we estimate the remaining elastic constant $C_{13}$ and the shape of the creep void by a micromechanics calculation, assuming that the voids are disk-shape ellipsoids and their minor axes are aligned along the loading direction.

The micromechanics calculation [11] was made on the basis of Eshelby's equivalentinclusion theory [12] and Mori-Tanaka mean-field theory [13]. We determined the aspect ratio of the void so as to obtain the best agreement between the calculated and measured elastic constants for the four measured $C_{i j}$. Concerning the volume fraction of the void, we adopted the porosity determined by the Archimedes method (TABLE I). The two isotropic 
elastic constants before the creep test were determined to be $C_{11}=199.8 \mathrm{GPa}$ and $C_{44}=47.06$ GPa by the pulse-echo method, which were input in the micromechanics calculation as the elastic constants of the isotropic matrix. Thus, the fitting parameter was only the aspect ratio.

The calculated and measured elastic constants are shown in TABLE II and compared in Fig.2. They favorably agree with each other, supporting the validity of the modeling with the disk-shape voids and the micromechanics calculation. The determined aspect ratio becomes larger as the creep damage progresses, indicating that creep voids preferentially grow and expand within the grain-boundary regions perpendicular to the loading direction. This is consistent with the observation by Morishita and Hirao [9]. Therefore, the creep voids on the grain boundaries can be considered as the principal cause of the softening and anisotropy of the macroscopic elastic constants.

Next, we discuss the local elastic stiffness. Figure 3 plots the histogram of the frequency (not the acoustical frequency but the normalized count) of the measured $\Delta f / f$ on the examined regions. The distributions are asymmetric because the defects decrease the effective elastic constant to yield low $\Delta f / f$ values. These distributions appear to obey the beta distribution

$$
\begin{aligned}
& F(x)=\frac{1}{B(\alpha, \beta)} x^{\alpha-1}(1-x)^{\beta-1} \\
& B(\alpha, \beta)=\int_{0}^{1} t^{\alpha-1}(1-t)^{\beta-1} d t
\end{aligned}
$$

Here, $F$ and $x$ correspond to the frequency (counts) and $\Delta f / f$, respectively. $\alpha$ and $\beta$ are fitting parameters. We fitted the beta function to the distribution and determined the 
expectation value (the average value) $(\Delta f / f)_{\text {ave }}$ by

$$
(\Delta f / f)_{a v e}=\frac{\alpha}{\alpha+\beta}
$$

TABLE III shows the average $\Delta f / f$ values for the six cases (two faces for each specimen).

In the previous study [3], we have established an analytical model to relate the resonancefrequency change of the oscillator to the effective elastic stiffness of the contacting anisotropic materials (see Appendix). This analysis allows us to convert a $\Delta f / f$ value into the effective normal modulus $E^{*}$.

Considering the elastic anisotropy, the effective normal modulus $E^{*}$ of $\mathrm{Cu}$ varies between 118 and $135 \mathrm{GPa}$ [3], depending on the crystallite orientation. (Note that this stiffness range is narrower than the range of Young's modulus, which varies between 65 and 190 GPa.) Figure 4 shows the relationship between the effective normal modulus and the frequency change calculated by the Lagrangian minimization with a Rayleigh-Ritz method [14], showing that the stiffness variation depending on the crystallite orientation will cause the variation of $\Delta f / f$ between $0.061 \%$ and $0.067 \%$. This range is shown by the vertical broken lines and the arrow in the top figure in Fig.3. For specimen \#1, all the measuring points show the frequency change larger than $0.04 \%$, and the major measuring points provide the frequency change within this range, indicating that the stiffness distribution is principally caused by the crystallite orientation. As the creep time increases, many measuring points show lowfrequency values out of this range; the average $\Delta f / f$ value decreases and very-low $\Delta f / f$ values appear, as seen in the inserts in Fig. 4. For example, the distribution on face A for specimen \#3 includes relatively higher count at $\Delta f / f=0.018 \%$, which corresponds to the 
effective normal modulus of $17 \mathrm{GPa}$ (smaller than the non-damaged value by a factor of 7 ). Thus, the local stiffness sensitively decreases reflecting the presence of defects, showing a great potential for a nondestructive evaluation in the field.

Finally, we discuss the relationship between the microscopic and macroscopic moduli. When a complete set of the elastic constants is known, the effective normal modulus along any direction can be obtained (Appendix). We thus calculated the effective normal modulus using the macroscopic five elastic constants (macroscopic moduli). The results are shown in TABLE III. They correlate with the average $\Delta f / f$ values measured by RUM (microscopic moduli), as shown in Fig. 5. Thus, the microscopic modulus qualitatively reflects the macroscopic modulus. For a quantitative comparison, we further calculated the possible frequency changes $(\Delta f / f)_{\text {cal }}$ using the effective normal modulus in TABLE III, and predicted the resonance-frequency change by the Rayleigh-Ritz method (Fig. 4). These values are based on the macroscopic stiffness and significantly exceed the measured frequency changes by RUM (microscopic values) for specimens $\# 2$ and $\# 3$ as seen in Fig. 5 . Therefore, the microscopic stiffness is lower than the macroscopic stiffness for specimens \#2 and \#3. This result is associated with the important observation that the resonance frequency decreases not only near the grain boundaries but also inside the grains as the creeping time increases (Fig. 1): The local stiffness inside a grain is affected. This result will be explained by the size effect: Vacancies and vacancy clusters will remain in the bulk as the creep time increases. In the RUM measurement, the effective volume near the contact interface, where the stress field caused by the biasing force becomes significant, is limited to only a few $\mu \mathrm{m}^{3}$. The stress 
field in such a localized region can increase the degree of the stress concentration near the defects there and causes larger deformation than do the plane-acoustic waves used in the pulse-echo measurements (wavelength is about $1 \mathrm{~mm}$ ). Therefore, the effective stiffness will decrease as the measuring volume becomes smaller provided that the defect size remains unchanged.

Finally we note that the RUM oscillator is easily installed in a cylindrical tube to make it mobile like a ball point pen for the use in the field, owing to its simple structure and the wireless-electrodeless measurement.

\section{CONCLUSION}

The resonance ultrasound microscopy showed a great potential for evaluating materials' local damages through the anomalous decrease of the local stiffness. It more clearly visualized the defects than did the optical microscopy, and it detected lowered stiffness regions where the optical microscopy did not observe any defects.

The macroscopic stiffness was evaluated by the pulse-echo method and by the micromechanics modeling. The microscopic stiffness was quantitatively analyzed by the contact theory for the contact between anisotropic bodies. The local-stiffness decrease occurred not only near the grain boundaries but also inside grains. The microscopic stiffness correlated with the macroscopic stiffness, but it showed much higher sensitivity to the defects. Thus, the resonance ultrasonic microscopy can be a powerful tool for the nondestructive evaluation of damaged solids. 


\section{REFERENCES}

[1] H. Ogi, J. Tian, T. Tada, and M. Hirao, "Elastic-stiffness mapping by resonance-ultrasound microscopy with isolated piezoelectric oscillator", Appl. Phys. Lett., vol. 83, pp. 464-466, 2003.

[2] J. Tian, H. Ogi, T. Tada, and M. Hirao, "Young's modulus mapping on SCS-6 SiC $f$ Ti-6Al-4V composite by electromagnetic-resonance-ultrasound microscopy", J. Appl. Phys., vol. 94, pp. 6472-6476, 2003.

[3] H. Ogi, M. Hirao, T. Tada, and J. Tian, "Elastic-stiffness distribution on polycrystalline copper studied by resonance ultrasound microscopy: Young's modulus microscopy", Phys. Rev. B, vol. 73, 174107, 2006.

[4] H. Ogi, H. Niho, and M. Hirao, "Elastic-Stiffness Distribution on Dual-Phase Stainless Steel Studied by Resonance Ultrasound Microscopy", Acta Materialia, 2006, in press.

[5] H. Ogi, H. Niho, and M. Hirao, "Internal-friction mapping on solids by resonance ultrasound microscopy", Appl. Phys. Lett., vol. 88, 141110, 2006.

[6] R. Truell, C. Elbaum, and B. Chick, Ultrasonic Methods in Solid Sate Physics (Academic Press, New York, 1969).

[7] H. Ogi, Y. Minami, and M. Hirao: " Acoustic study of dislocation rearrangement at later stages of fatigue: Non-contact prediction of remaining life", J. Appl. Phys., vol. 90, pp. 438$442,2002$.

[8] T. Ohtani, H. Ogi and M. Hirao, "Evolution of Microstructure and Acoustic Damping during Creep of a Cr-Mo-V Ferritic Steel", Acta Materialia, vol. 54, pp. 2705-2713, 2006.

[9] T. Morishita and M. Hirao, "Creep damage modeling based on ultrasonic velocities in copper", Int. J. Sol. Struc., vol. 34, pp. 1169-1182, 1997.

[10] M. Hirao, H. Ogi, N. Suzuki, and T. Ohtani "Ultrasonic attenuation peak during fatigue of polycrystalline copper", Acta Materialia, vol. 48, pp. 517-524, 2000.

[11] H. Ogi, G. Shimoike, M. Hirao, K. Takashima, and Y. Higo, "Anisotropic elastic-stiffness coefficients of an amorphous Ni-P film", J. Appl. Phys., vol. 91, pp. 4857-4862, 2002.

[12] J. D. Eshelby, "The determination of the elastic field of an ellipsoidal inclusion and related problems, Proc. Roy. Soc. London, A, vol. 241, pp. 376-396, 1957. 
[13] T. Mori and K. Tanaka, "Average stress in matrix and average elastic energy of materials with misfitting inclusions", Acta Metall., vol. 21, pp. 571-574, 1973.

[14] H. Ogi, T. Tada, J. Tian, and M. Hirao, "Resonant ultrasound microscopy with an isolated langasite oscillator for quantitative evaluation of local elastic constant", Jpn. J. Appl. Phys., vol. 44, pp. 4381-4384 2005.

[15] J. Willis, "Hertzian contact of anisotropic bodies", J. Mech. Phys. Sol., vol. 14, pp. 163-176, 1966.

[16] S. Swanson, "Hertzian contact of orthotropic materials", Int. J. Sol. Struc., vol. 41, pp. 1945$1959,2004$.

[17] G. Yaralioglu, F. Degertekin, K. Crozier, and C. Quate, "Contact stiffness of layered materials for ultrasonic atomic force microscopy", J. Appl. Phys., vol. 87, pp. 7491-7496, 2000. 


\section{APPENDIX. Effective Normal Stiffness for the Contact between Anisotropic Bod-} ies

The oscillator's resonance frequency can be accurately calculated by the Lagrangian minimization with the Rayleigh-Ritz method, when the contact stiffness at the contact interface is given [14]. Willis [15] analyzed the elastic field caused by the contacts between two anisotropic bodies using Fourier transform, and Swanson [16] derived the indentation depth $\delta$ following Willis's approach in the case of the contact with orthorhombic materials when the orthorhombic axes are parallel to the contact surface. The result is given by

$$
\delta=\frac{I_{3}}{4}\left(\frac{9 F_{0}^{2}}{2 R I_{1}}\right)^{\frac{1}{3}}
$$

Where $F_{0}$ is the biasing force normal to the contact surface, and $R$ denotes the tip radius. $I_{1}$ and $I_{3}$ are obtained by integrating the Green function for a point-force indentation, which depends on the anisotropic elastic constants of the specimen and tip [16].

Harmonic oscillation of the oscillator causes harmonic perturbation in the force and the total force will be of the form $F_{0}+\Delta F$ at the contact interface. We assume $|\Delta F|<<\left|F_{0}\right|$ to obtain $\Delta \delta \approx \Delta F\left(I_{3}^{3} / 48 F_{0} R I_{1}\right)^{1 / 3}$. Thus, we define

$$
k=\sqrt[3]{\frac{48 F_{0} R I_{1}}{I_{3}^{3}}}
$$

as the contact stiffness at the interface. Classical Hertzian theory has related the effective modulus $E^{*}$ to the contact stiffness $k$ through $E^{*}=\sqrt{k^{3} /\left(6 R F_{0}\right)}$ in the case of contact of two isotropic bodies [17]. Similarly, we define the effective normal modulus $E^{*}$ for the 
contact between the anisotropic bodies by

$$
E^{*}=\sqrt{\frac{8 I_{1}}{I_{3}^{3}}}
$$

Thus, $E^{*}$ of the specimen is obtained by calculating $I_{1}$ and $I_{3}$ numerically, when the biasing force $F_{0}$ and the tip radius $R$ are given. The biasing force is unambiguously determined from the weight of the probe $[3,5]$. We determined the tip radius by fitting the calculated effective modulus to the one determined from the macroscopic elastic constants of a non-damaged copper specimen. (The tip radius was $0.48 \mathrm{~mm}$ ). 
TABLE I: Creep time and porosity of the specimens.

\begin{tabular}{lcc}
\hline \hline & creep time $(\mathrm{h})$ & porosity $\left(10^{-3}\right)$ \\
\hline specimen \#1 & 59.8 & 1.06 \\
specimen \#2 & 298.9 & 6.18 \\
specimen \#3 & 538.0 & 20.0 \\
\hline \hline
\end{tabular}


TABLE II: Elastic constants $C_{i j}(\mathrm{GPa})$ measured by the pulse-echo method and those calculated by the micromechanics calculation, and the aspect ratio of the creep void determined inversely by the micromechanics calculation.

\begin{tabular}{|c|c|c|c|c|c|c|c|c|c|}
\hline & \multicolumn{3}{|c|}{ specimen \#1 } & \multicolumn{3}{|c|}{ specimen \#2 } & \multicolumn{3}{|c|}{ specimen \#3 } \\
\hline & meas. & calc. & diff. $(\%)$ & meas. & calc. & diff. (\%) & meas. & calc. & diff. $(\%)$ \\
\hline$C_{11}$ & 199.45 & 199.62 & -0.08 & 196.89 & 197.90 & -0.51 & 181.22 & 186.91 & -3.14 \\
\hline$C_{33}$ & 199.35 & 199.46 & -0.06 & 195.44 & 194.85 & 0.30 & 165.92 & 162.32 & 2.17 \\
\hline$C_{44}$ & 47.00 & 47.02 & -0.04 & 46.48 & 46.76 & -0.61 & 43.40 & 45.10 & -3.93 \\
\hline$C_{66}$ & 47.01 & 46.88 & 0.26 & 46.63 & 46.30 & 0.71 & 45.18 & 45.73 & -1.22 \\
\hline$C_{12}{ }^{a}$ & 105.43 & 105.85 & -0.40 & 103.62 & 101.04 & 2.48 & 90.76 & 95.46 & -5.17 \\
\hline$C_{13}^{b}$ & - & 105.13 & - & - & 101.04 & - & - & 85.92 & - \\
\hline pect ratio ${ }^{b}$ & & 1.76 & & & 2.04 & & & 4.22 & \\
\hline
\end{tabular}

${ }^{a} C_{12}=C_{11}-2 C_{66}$

${ }^{b}$ determined by the micromechanics calculation 
TABLE III: The effective normal moduli $E^{*}(\mathrm{GPa})$ on faces $\mathrm{A}$ and $\mathrm{B}$ determined by the macroscopic elastic constants; and predicted $\left((\Delta f / f)_{\text {calc }}\right)$ and measured $\left((\Delta f / f)_{\text {ave }}\right)$ average resonance-frequency changes.

\begin{tabular}{ccccc}
\hline \hline & specimen & $E^{*}$ & $(\Delta f / f)_{\text {calc }}(\%)$ & $(\Delta f / f)_{\text {ave }}(\%)$ \\
\hline \multirow{2}{*}{ face A } & $\# 1$ & 127.5 & 0.0642 & 0.0642 \\
& $\# 2$ & 126.8 & 0.064 & 0.0586 \\
& $\# 3$ & 115.3 & 0.06 & 0.0541 \\
\hline \multirow{2}{*}{ face B } & $\# 1$ & 127.4 & 0.0641 & 0.063 \\
& $\# 2$ & 126.9 & 0.064 & 0.0593 \\
& $\# 3$ & 123.1 & 0.0627 & 0.0567 \\
\hline \hline
\end{tabular}




\section{Figure Caption}

Fig. 1 Microstructures on(a)face A of specimens \#1, (b)face B of specimen \#2, and (c)face B of specimen \#3, respectively, observed by optical microscopy; and corresponding stiffness images below them, (d), (e), and (f), measured by RUM. Small resonancefrequency-change values, indicating defects, are shown in the stiffness images. Dashed lines indicate grain boundaries. The loading direction is the horizontal direction.

Fig. 2 Elastic constants measured by the pulse-echo method and those calculated by the micromechanics modeling. The slope of the broken line is 1 .

Fig. 3 Histograms of the frequency (counts) of the resonance-frequency change on the examined regions for specimens $\# 1, \# 2$, and \#3. The white line expresses the fitted beta function. The vertical broken lines and the arrow in the top figure show the possible resonance-frequency changes caused by the orientation of the grains. The inserts highlight relatively higher counts of low resonance-frequency-change values.

Fig. 4 Relationship between the resonance-frequency change and the effective normal Young's modulus $E_{\text {aniso }}$.

Fig. 5 Comparison of the average resonance-frequency change measured by RUM (i.e. the microscopic value) with that predicted by the macroscopic elastic constants and the effective normal modulus determined by the microscopic elastic constants. The slope of the broken line is 1 , given for showing the lower measured frequency change for specimens \#2 and \#3. 


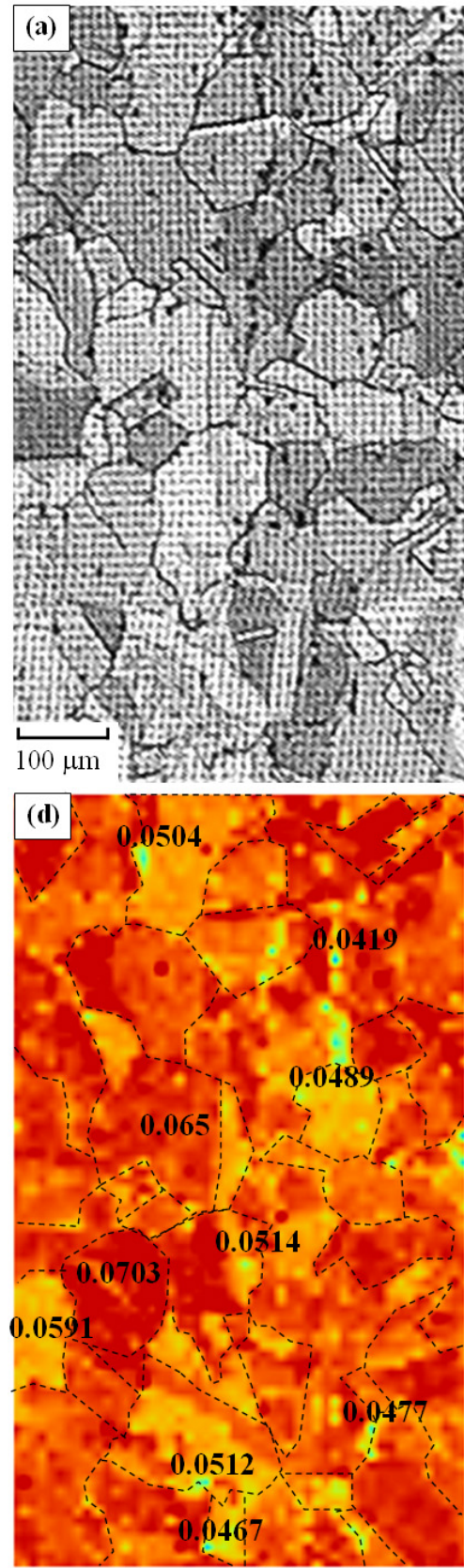

Specimen \#1 (face A)
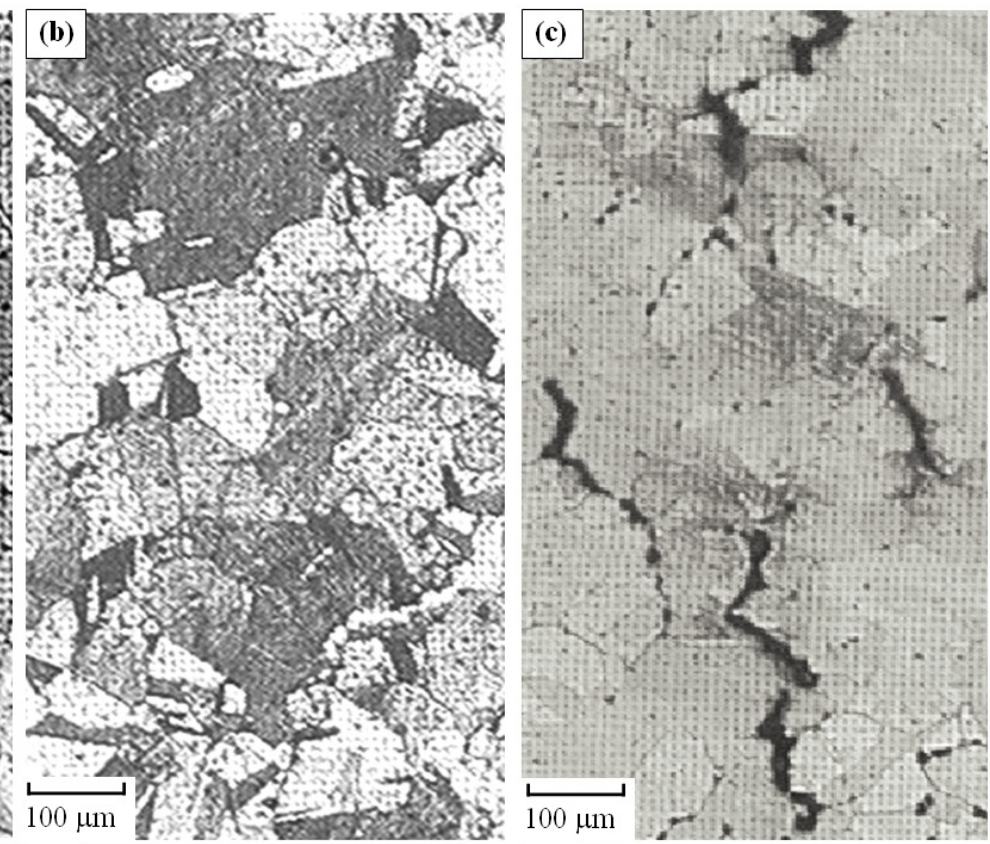

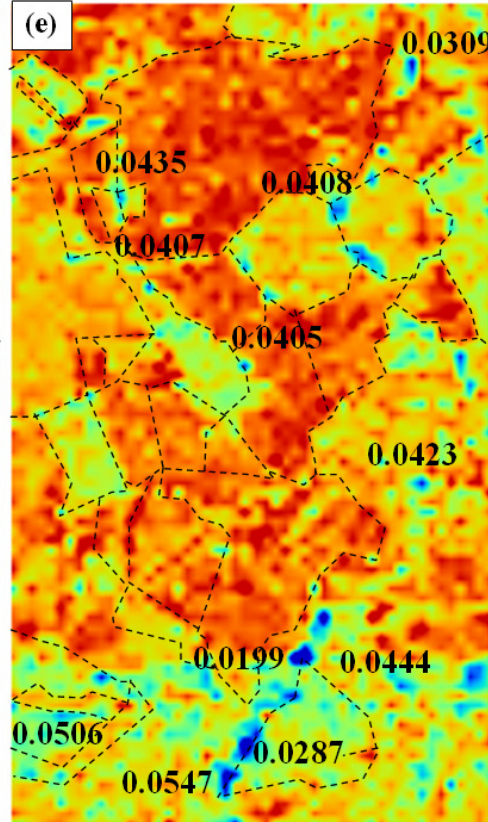

Specimen \#2 (face B)

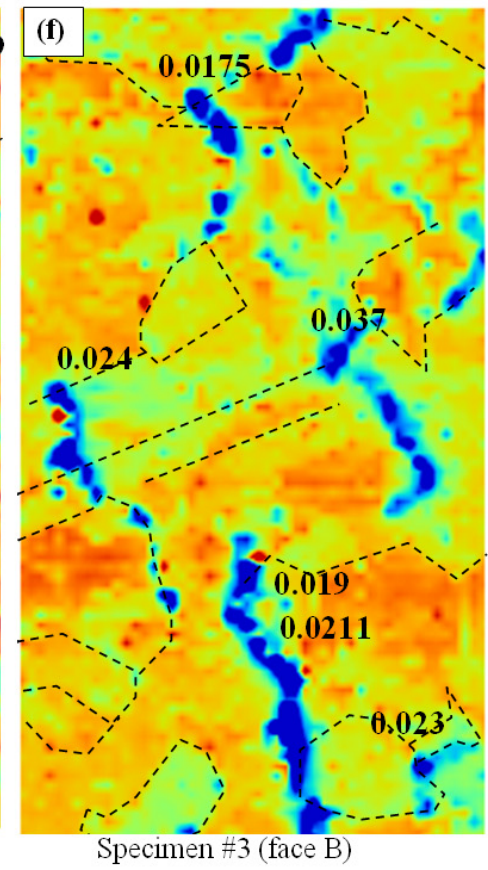

Specimen \#3 (face B)

$0.035 \quad \Delta f / f \quad 0.068(\%)$

FIG. 1: 


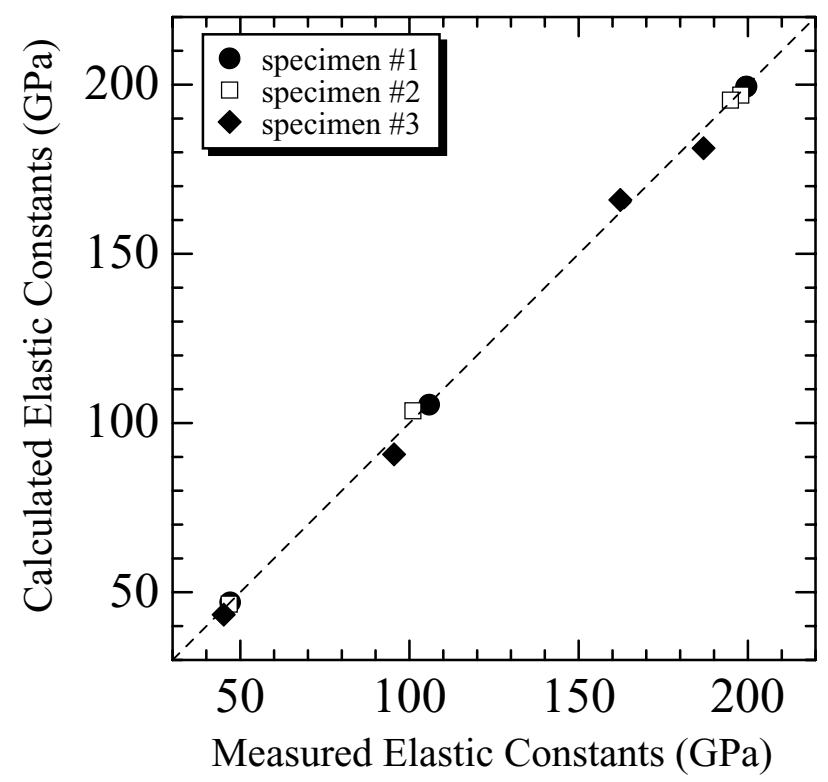

FIG. 2: 


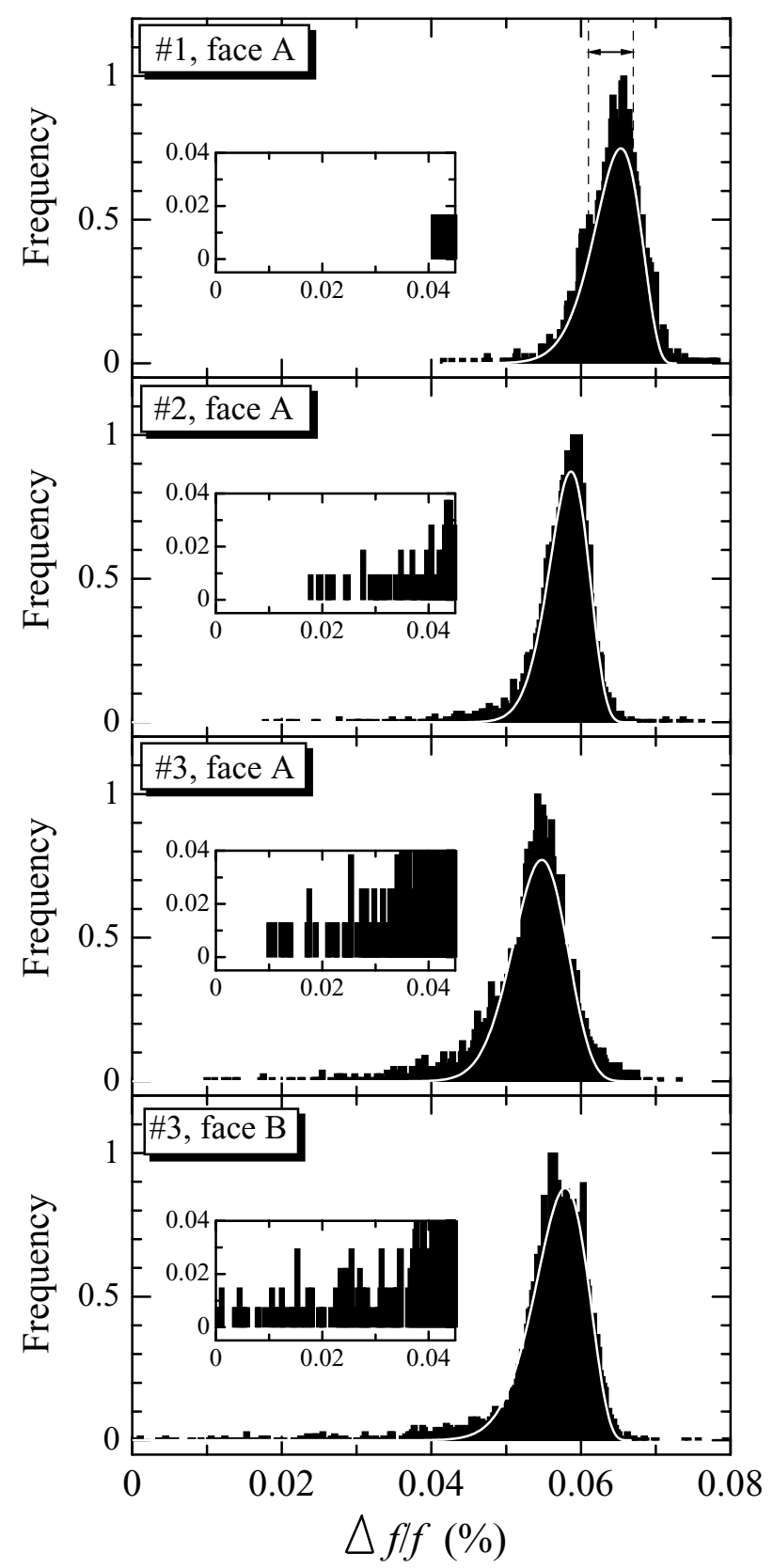

FIG. 3: 


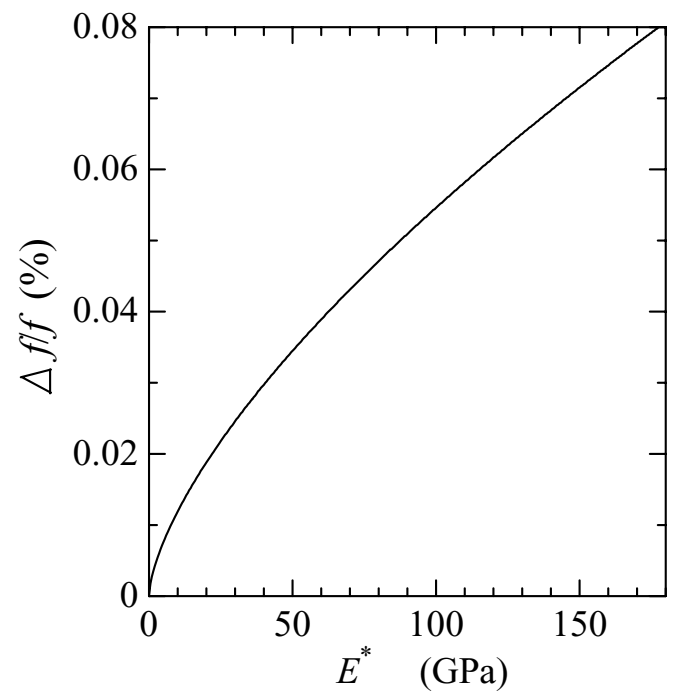

FIG. 4: 


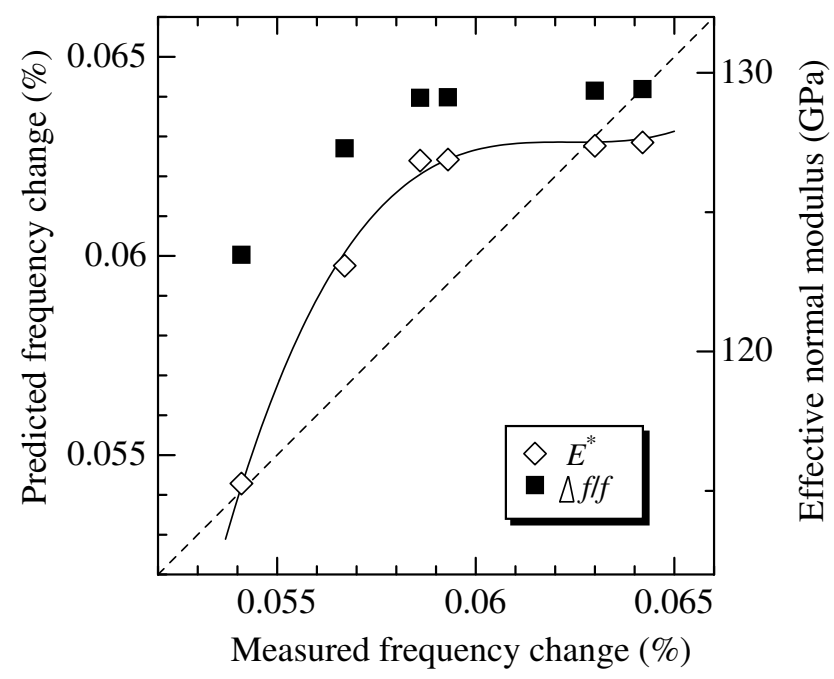

FIG. 5: 